\title{
Epileptic seizures in the emergency room: clinical and electroencephalographic findings associated with brain perfusion patterns on computed tomography
}

\author{
$J_{L}$ Restrepo-Vera ${ }^{1}$ (I) P. Coscojuela ${ }^{2} \cdot$ E. Fonseca ${ }^{3}$ (D) M. Quintana ${ }^{3} \cdot$ S. Sarria-Estrada ${ }^{2} \cdot$ E. Santamarina ${ }^{3} \cdot$ L. Abraira $^{3}$.

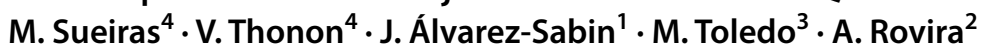

Received: 17 November 2021 / Revised: 29 January 2022 / Accepted: 31 January 2022 / Published online: 13 February 2022

(C) The Author(s), under exclusive licence to Springer-Verlag GmbH Germany 2022

\begin{abstract}
Background Diagnosis of epileptic seizures, particularly regarding status epilepticus (SE), may be challenging in an emergency room setting. The aim of the study was to study the diagnostic yield of perfusion computed tomography (pCT) in patients with single epileptic seizures and SE.

Methods We retrospectively reviewed the records of patients who followed an acute ischemic stroke pathway during a 9-month period and who were finally diagnosed with a single epileptic seizure or SE. Perfusion maps were visually analyzed for the presence of hyperperfusion and hypoperfusion. Clinical data, EEG patterns, and neuroimaging findings were compared.

Results We included 47 patients: 20 (42.5\%) with SE and 27 (57.5\%) with single epileptic seizure. Of 18 patients who showed hyperperfusion on pCT, 12 were ultimately diagnosed with SE and eight had EEG findings compatible with an SE pattern. Focal hyperperfusion on pCT had a sensitivity of 60\% (95\% CI 36.4-80.2) and a specificity of 77.8\% (95\% CI 57.2-90.6) for predicting a final diagnosis of SE. The presence of cerebral cortical and thalamic hyperperfusion had a high specificity for predicting SE presence. Of note, $96 \%$ of patients without hyperperfusion on pCT did not show an SE pattern on early EEG. Conclusions In acute settings, detection by visual analysis of focal cerebral cortical hyperperfusion on pCT in patients with epileptic seizures, especially if accompanied by the highly specific feature of thalamic hyperperfusion, is suggestive of a diagnosis of SE and requires clinical and EEG confirmation. The absence of focal hyperperfusion makes a diagnosis of SE unlikely.
\end{abstract}

Keywords Computed tomography $\cdot$ Epilepsy $\cdot$ Neuroimaging $\cdot$ Perfusion $\cdot$ Seizures $\cdot$ Status epilepticus $\cdot$ Thalamus

E. Fonseca

e.fonseca@vhebron.net

1 Neurology Department, Vall d'Hebron University Hospital, Medicine Department, Universitat Autònoma de Barcelona, Passeig Vall d'Hebron 119-129, 08035 Barcelona, Spain

2 Neuroradiology Section, Radiology Department, Vall d'Hebron University Hospital, Vall d'Hebron Research Institute, Passeig Vall d'Hebron 119-129, 08035 Barcelona, Spain

3 Epilepsy Unit, Neurology Department, Vall d'Hebron University Hospital, Medicine Department, Universitat Autònoma de Barcelona, Passeig Vall d'Hebron 119-129, 08035 Barcelona, Spain

4 Neurophysiology Department, Vall d'Hebron University Hospital, Universitat Autònoma de Barcelona, Passeig Vall d'Hebron 119-129, 08035 Barcelona, Spain

\section{Introduction}

Epileptic seizures are a common reason for emergency room (ER) visits [1]. Status epilepticus (SE), the most severe form of epilepsy, is a life-threatening condition [2]. Its incidence is rising and is currently estimated at approximately 10-20 cases per 100,000 person-years [3]. Diagnosis of SE is challenging and requires clinical experience and paraclinical diagnostic tools. Stroke and post-ictal epileptic phenomena often mimic SE without prominent motor symptoms (nonconvulsive SE [NCSE]) [4]. In such cases, an electroencephalogram (EEG) is necessary to confirm diagnosis, but even at tertiary hospitals that deal with neurological emergencies, access to EEG can be delayed by $4 \mathrm{~h}$ or longer [5]. Seizure duration is one of the main modifiable prognostic factors in 
$\mathrm{SE}$ and reductions in this time are closely correlated with better outcomes [6, 7].

Brain perfusion computed tomography $(\mathrm{pCT})$ is a widely used neuroradiological imaging modality in ERs [8], and previous studies have shown its diagnostic value in patients with acute neurological deficits of epileptic origin [9-11]. Ictal abnormalities seen on pCT have several explanations, including upregulation of the neurovascular unit and blood-brain barrier disruption mediated by the hyperglutamatergic state of the brain during SE [12,13]. There is thus growing interest in $\mathrm{pCT}$ as a potential tool for the rapid identification of epileptic seizures and SE in particular. The aim of this study was to assess the diagnostic yield of emergency pCT in single epileptic seizures and SE in an ER setting.

\section{Methods}

This was a single-center retrospective study of patients aged $\geq 18$ years who underwent pCT following a noncontrast CT and CT angiography of the brain for suspected acute stroke within $24 \mathrm{~h}$ of symptom onset in the ER and who were subsequently diagnosed with a single epileptic seizure or SE following additional tests. The study was conducted in a tertiary hospital that offers emergency EEG and $\mathrm{pCT}$ and was approved by the local ethics committee (PR(AG)285/201). Data from all patients who followed the hospital's acute stroke care pathway at any time between November 2019 and July 2020 were systematically obtained from the hospital's electronic database. Those with unclear seizures, a new ischemic or hemorrhagic lesion on brain CT or magnetic resonance imaging (MRI), or cerebral arterial occlusion or stenosis on angiographic sequences were excluded. All patients were assessed by two neurologists on arrival at the ER. Epileptic seizures were diagnosed based on clinical features and EEG findings where available. SE was diagnosed based on clinical semiology and the EEG criteria from the 2015 Report of the International League Against Epilepsy Task Force on the Classification of Status Epilepticus [14]. Hyperperfusion on pCT was not taken into account to establish the diagnosis of SE. Clinical, EEG, and imaging data were retrospectively collected from the hospital's electronic database. A record was also made of all intravenous anti-seizure drugs (ASDs) administered at any time between symptom onset and ancillary tests.

All patients underwent non-contrast CT, CT angiography, and $\mathrm{pCT}$ on a 128-slice scanner (Somatom Definition, Siemens Healthcare). The pCT study included dynamic acquisition $(70 \mathrm{kV}$ and $150 \mathrm{mAs})$ with injection of $50 \mathrm{~mL}$ of nonionic iodinated contrast material (Iohexol) at a concentration of $350 \mathrm{mg} / \mathrm{mL}$ (Omnipaque 350) using an injection pump to obtain a constant flow of $5 \mathrm{~mL} / \mathrm{s}$. The acquisition conditions in all the imaging modalities were a slice thickness of $5 \mathrm{~mm}$, a rotation time of $0.28 \mathrm{~s}$, an image matrix of $512 \times 512$ to the convexity, and collimation of $32 \times 1.2 \mathrm{~mm}$. In total, 700 images were obtained per patient and processed at a workstation to analyze cerebral blood volume (CBV), regional cerebral blood flow volume (CBF), time to peak (TTP), and mean transit time (MTT). The images were reassessed by an experienced neuroradiologist (P.C.) blinded to the clinical data including final diagnosis and the findings of EEG. A qualitative visual analysis was carried out to identify areas showing perfusion differences in the various hemodynamic maps, focusing on cerebral cortex and thalamus. For the visual inspection of the raw $\mathrm{pCT}$ images, hyperperfusion was defined as an increase in CBF and a decrease in TTP and MTT in cerebral cortex and/or thalamus. Hypoperfusion was defined as focally decreased CBF and increased TTP and MTT relative to the contralateral brain hemisphere in all cases.

Emergent EEG lasting $\geq 45$ min was performed within $24 \mathrm{~h}$ of symptom onset. All EEGs were recorded on a 32and/or 64-channel digital EEG system (XLTEK, Deltamed). An array of 21 electrodes was used beyond the 10-20 system. The combination of referential (average) and bipolar EEG derivations with other signals (ECG and EMG) allowed for the acquisition of polygraphic EEG recordings. These were evaluated by an expert neurophysiologist (M.S. or V.T.), blinded to the pCT images. The EEG findings were described using the standardized international nomenclature of the International Federation of Clinical Neurophysiology and the American Clinical Neurophysiology Society's standardized terminology for neurocritical care [15]. The Salzburg criteria were used for the diagnosis of SE patterns on EEG [16]. Specific EEG features were categorized as nonepileptiform abnormalities (NEA), interictal epileptiform discharges (IED), single epileptic seizures (absence of continuous or fluctuating patterns suggestive of SE), and EEG patterns consistent with SE.

Descriptive and frequency statistics were obtained, and comparisons made in IBM SPSS Statistics, version 22.0. Comparisons between the three perfusion pattern groups (hyperperfusion, hypoperfusion, normal perfusion) were made using the Fisher exact test for categorical variables and the Kruskal-Wallis test for numerical variables. Significance was set at $p<0.05$. Sensitivity, specificity, and positive and negative predictive values were calculated to assess the diagnostic value of $\mathrm{pCT}$ relative to final diagnosis and EEG findings.

\section{Results}

Of the 697 patients assessed during the study period, 47 $(6.74 \%)$ met the inclusion criteria. The selection process and results of the imaging tests are shown in Fig. 1. 


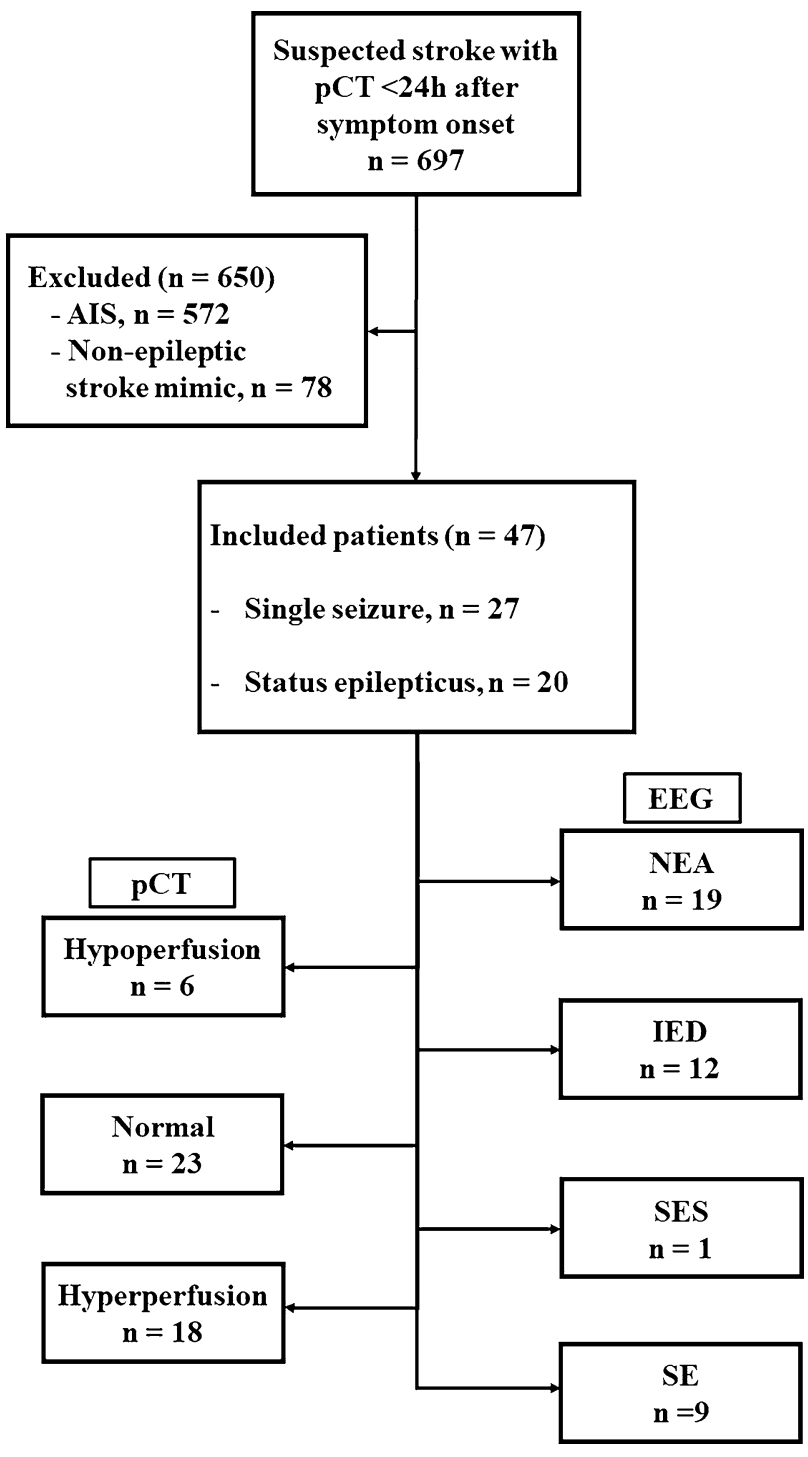

Fig. 1 Flow chart of patient inclusion/exclusion process and pCT/ EEG results. pCT, perfusion computed tomography; AIS, acute ischemic stroke; EEG, electroencephalogram; NEA, non-epileptic abnormalities; IED, interictal epileptiform discharges; SES, single epileptic seizure; SE, status epilepticus

The mean age of the patients was $72.8 \pm 16.1$ years and 26 (55.3\%) were women. Twenty-five patients (53.2\%) underwent a brain MRI, while in the remaining 22 (46.8\%), no follow-up imaging was considered necessary to clarify the diagnosis by the treating physician. Twenty patients $(42.5 \%)$ received a final diagnosis of SE: nine (45\%) patients suffered from NCSE with or without coma along with EEG patterns consistent with SE and 11 (55\%) patients were clinically diagnosed with SE with prominent motor symptoms; 10 of these patients had a non-ictal pattern on EEG and one died shortly after CT before EEG could be performed due to concurrent fatal pneumonia from SARS-CoV-2 infection. The remaining 27 patients
(57.5\%) of the sample received a final diagnosis of a single epileptic seizure. No seizure clusters were observed. The clinical characteristics of the patients and EEG/pCT findings are summarized in Table 1.

Brain $\mathrm{pCT}$ was performed a median of $3 \mathrm{~h}$ (interquartile range [IQR], 2-12 h) after the last time seen asymptomatic. The scan was abnormal in 24 patients (51\%): focal hyperfusion (cerebral cortical hyperperfusion accompanied or not by thalamic hyperperfusion) in 18 cases (38.3\%) and focal hypoperfusion in six (12.7\%). Thalamic hyperperfusion was identified in $8(17 \%)$ of 47 patients, all of them with concurrent homolateral cortical hyperperfusion. No thalamic hypoperfusion was detected. Of the 18 patients with hyperperfusion, $12(66.7 \%)$ were ultimately diagnosed with SE and six (33.3\%) with a single epileptic seizure (Fig. 2a). All the patients in the hypoperfusion group were diagnosed with a single epileptic seizure. Three (50\%) of them did not undergo brain MRI but previous diagnosis of epilepsy in all of them, no evidence of acute lesion on CT and transient deficits were considered sufficient evidence to reasonably rule out a transient ischemic stroke. The frequency of pCT alterations was not significantly influenced by time from symptom onset to $\mathrm{pCT}$, seizure etiology, or intravenous administration of ASDs before neuroimaging studies (Table 1). In most cases, hyperperfusion was exclusively observed in the cortical gray matter, accompanied or not of homolateral thalamic hyperperfusion, with relative preservation of the underlying white matter (Fig. 3). Most cases of focal hypoperfusion involved both the cortical and subcortical regions.

EEG was performed in 41 patients $(87.2 \%)$. Median time to EEG was $16 \mathrm{~h}$ (IQR, 8.5-22.5 h) from last time seen asymptomatic and $8 \mathrm{~h}$ (IQR: 4-14.5) from pCT. EEG showed epileptiform abnormalities in 22/41 patients (53.7\%): IEDs in 12, EEG patterns consistent with SE in nine, and a single epileptic seizure in one. Eight of the nine patients with EEG findings consistent with SE showed hyperfusion on the admission pCT. Patients with other EEG abnormalities were more likely to show normal perfusion (Fig. 2a).

Detection of thalamic hyperperfusion accompanying cortical cerebral hyperperfusion was significantly associated to $\mathrm{SE}$ as final diagnosis and to an SE pattern on urgent EEG (Table 2).

Hyperperfusion on pCT had a sensitivity of $60 \%$ (95\% CI, 36.4-80.2) for the detection of SE as final diagnosis. Thalamic hyperperfusion on $\mathrm{pCT}$ was identified exclusively in patients with final diagnosis of SE. The rest of the corresponding values for predicting final diagnosis or the presence of EEG patterns consistent with SE are shown in Table 3. Of note, $96 \%$ of patients without hyperperfusion on pCT did not have an SE pattern on EEG. 
Table 1 Demographic, clinical, and EEG features according to pCT findings

\begin{tabular}{|c|c|c|c|c|c|}
\hline \multirow[t]{2}{*}{ Demographic, clinical, and EEG findings } & \multicolumn{5}{|l|}{ pCT findings } \\
\hline & Total $(n=47)$ & $\begin{array}{l}\text { Hypoperfusion } \\
(n=6,12.7 \%)\end{array}$ & $\begin{array}{l}\text { Normal } \\
(n=23 ; 48.9 \%)\end{array}$ & $\begin{array}{l}\text { Hyperperfusion } \\
(n=18 ; 38.3 \%)\end{array}$ & $P$ value \\
\hline Age; years $($ mean $\pm \mathrm{SD})$ & $72.8(16.1)$ & $72(15.1)$ & $78.4(15.2)$ & $65.5(15.3)$ & $0.046^{*}$ \\
\hline \multicolumn{6}{|l|}{ Sex } \\
\hline Female; $n(\%)$ & $26(55.3)$ & $6(100)$ & $13(56.5)$ & $7(38.9)$ & \multirow[t]{2}{*}{$\mathbf{0 . 0 2 7}$} \\
\hline Male; $n(\%)$ & $21(44.7)$ & $0(0)$ & $10(44.5)$ & $11(61.1)$ & \\
\hline \multicolumn{6}{|l|}{ History of epilepsy } \\
\hline Yes; $n(\%)$ & $11(23.4)$ & $3(50)$ & $2(8.7)$ & $6(33.3)$ & \multirow[t]{2}{*}{0.047} \\
\hline No; $n(\%)$ & $36(76.6)$ & $3(50)$ & $21(91.3)$ & $12(66.6)$ & \\
\hline Time from onset to pCT; hours (median, IQR) & $3(2-12)$ & $7.5(1-14.7)$ & $3(2-13)$ & $4(1.2-7.5)$ & 0.9 \\
\hline Time from onset to EEG ${ }^{*}$; hours (median, IQR) & $16(8.5-22.5)$ & $15(10.7-19.2)$ & $16(7.5-23.5)$ & $15(9.7-21.7)$ & 0.99 \\
\hline \multicolumn{6}{|l|}{ Diagnosis } \\
\hline $\mathrm{SS} ; n(\%)$ & $27(57.4)$ & $6(100)$ & $15(65.2)$ & $6(33.3)$ & \multirow[t]{2}{*}{0.01} \\
\hline $\mathrm{SE} ; n(\%)$ & $20(42.6)$ & $0(0)$ & $8(34.8)$ & $12(66.7)$ & \\
\hline \multicolumn{6}{|l|}{ Seizure etiology } \\
\hline Metabolic; $n(\%)$ & $10(21.3 \%)$ & $1(16.7)$ & $3(13)$ & $6(33.3)$ & \multirow[t]{6}{*}{0.607} \\
\hline Vascular; $n(\%)$ & $10(21.3 \%)$ & $1(16.7)$ & $4(17.4)$ & $5(27.8)$ & \\
\hline Tumoral; $n(\%)$ & $9(19.1)$ & $1(16.7)$ & $5(21.7)$ & $3(16.7)$ & \\
\hline ASD withdrawal; $n(\%)$ & $3(6.4)$ & $1(16.7)$ & $1(4.3)$ & $1(5.6)$ & \\
\hline PRES; $n(\%)$ & $3(6.4)$ & $1(16.7)$ & $2(8.7))$ & $0(0)$ & \\
\hline Unknown; $n(\%)$ & $12(25.5)$ & $1(16.7)$ & $8(34.8 \%)$ & $3(16.7)$ & \\
\hline \multicolumn{6}{|l|}{ ASDs before pCT } \\
\hline Yes; $n(\%)$ & $15(31.9)$ & $3(50)$ & $9(39.1)$ & $3(16.7)$ & \multirow[t]{2}{*}{0.194} \\
\hline No; $n(\%)$ & $32(68.1)$ & $3(50)$ & $14(60.9)$ & $15(83.3)$ & \\
\hline \multicolumn{6}{|l|}{$\mathrm{EEG}^{\ddagger}$} \\
\hline $\mathrm{NEA} ; n(\%)$ & $19(46.3)$ & $3(75)$ & $13(61.9)$ & $3(18.8)$ & \multirow[t]{4}{*}{0.008} \\
\hline IED; $n(\%)$ & $12(29.3)$ & $1(25)$ & $6(28.6)$ & $5(31.3)$ & \\
\hline $\mathrm{SES} ; n(\%)$ & $1(2.4)$ & $0(0)$ & $1(4.8)$ & $0(0)$ & \\
\hline $\mathrm{SE} ; n(\%)$ & $9(22)$ & $0(0)$ & $1(4.8)$ & $8(50)$ & \\
\hline
\end{tabular}

$E E G$ electroencephalography, $p C T$ perfusion computed tomography, $S D$ standard deviation, $I Q R$ interquartile range, $S S$ singles seizure, $S E$ status epilepticus, $A S D s$ anti-seizure drugs, $P R E S$ posterior reversible encephalopathy syndrome, $N E A$ non-epileptiform abnormalities, $I E D$ interictal epileptiform discharges, $S E S$ single epileptic seizure

*Bold typed $p$ values represent statistically significant differences between groups.

\$: 41 patients underwent EEG registry. Percentages are calculated according to these numbers.

\section{Discussion}

In the present study of patients presenting with an epileptic seizure at the ER of a tertiary hospital, focal hyperperfusion on early pCT was a useful marker of SE but not single epileptic seizures. Nearly two-thirds of the patients ultimately diagnosed with SE showed focal hyperperfusion on $\mathrm{pCT}$, while those diagnosed with a single epileptic seizure mostly showed normal brain perfusion. These findings are consistent with previous reports of brain hemodynamics during SE and post-ictal states in similar settings [17].

However, a precise relationship between diagnosis of SE and pCT pattern was not observed in our study. Hyperperfusion had a $60 \%$ sensitivity and a $77.8 \%$ specificity for the detection of SE as a final diagnosis; this diagnostic yield is similar to that described in retrospective studies with a comparable median time from pCT to EEG (e.g., $8 \mathrm{~h}$ or more) $[10,11,18]$. Simultaneous acquisitions of EEG and pCT that could not only study the perfusion signature of SE but also characterize the observed brain perfusion changes depending on the EEG pattern, frequency and/or morphology of the observed ictal discharges or rhythmic activity could provide greater insights into why over one-third of the patients diagnosed with SE showed no abnormalities on pCT on our study. The authors of a retrospective study of seven critically ill patients who underwent fluorodeoxyglucose-positron emission tomography during EEG monitoring observed a positive correlation between the frequency of 


\section{A. Final Diagnosis}

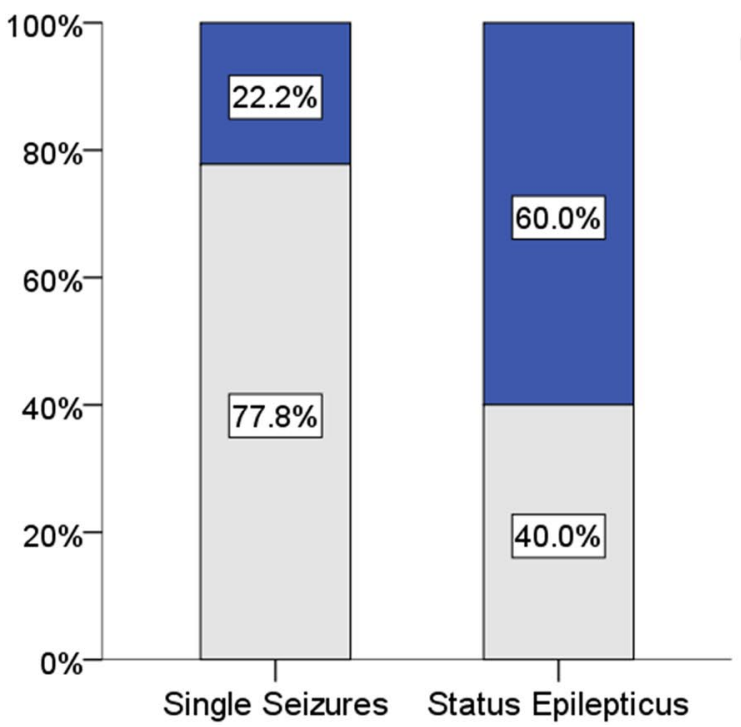

Fig. 2 Cumulative proportion of patients showing hyperperfusion on pCT according to final diagnosis (A) and EEG patterns (B). The bars represent groups of patients. Approximately two of every three patients who received a final diagnosis of SE had hyperperfusion. In the group of patients diagnosed with a single epileptic seizure, $78 \%$ showed no pCT abnormalities. EEG findings compatible with an SE
B. EEG findings

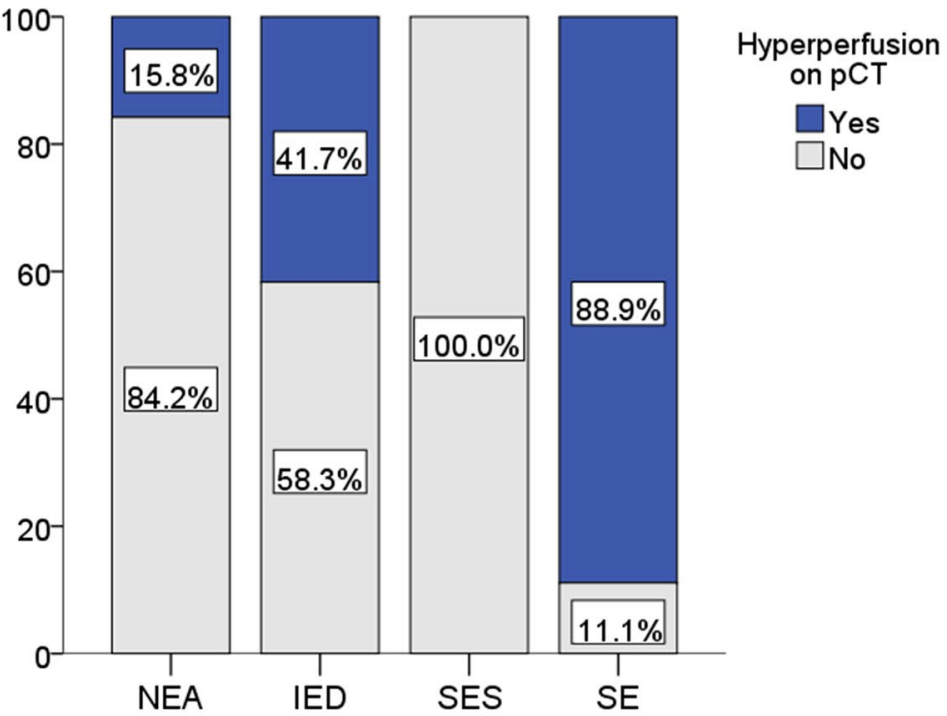

lateralized periodic discharges and glucose metabolism [19]. Taking in mind the unfeasibility of the latter in an ER setting, our group[9] compared prospectively in a previous study pCT images of patients with an EEG-confirmed diagnosis of focal-onset SE with post-ictal control patients and found that pCT hyperperfusion identified four of the five confirmed SE episodes. Similar results were reported by Hauf et al. [20] in a retrospective study of patients with confirmed NCSE who had previously undergone urgent pCT. Thus, although its presence is significantly suggestive of SE diagnosis when compared to single epileptic seizures in the proper setting, the low detection rate for SE diagnosis obtained in the present study underlines the in-force preponderance of clinical assessment and EEG over pCT examination in the ER. On the other hand, these results underscore the dynamic nature of hemodynamic perfusion changes associated with SE, previously demonstrated by human single-photon emission computed tomography [21].

However, the findings of the present study also point to a significant relationship between focal hyperperfusion of the brain measurable by $\mathrm{pCT}$ and epileptiform discharges on EEG in an acute setting, particularly in SE and sustained ictal activity on EEG. In a smaller study, Payabvash et al. [23] found that patients with epileptiform interictal discharges on follow-up EEG within 1 week of pCT showed pattern were almost exclusively observed in patients with hyperperfusion on pCT and more than two-fifths of patients with IEDs had hyperperfusion. pCT, perfusion computed tomography; NEA, nonepileptiform abnormalities; IED, interictal epileptiform discharges; SES, single epileptic seizure; SE, status epilepticus

higher ipsilateral relative $\mathrm{CBF}$ and $\mathrm{CBV}$ than patients with continuous or polymorphic slowing and normal EEG findings. In our study, however, most patients benefited from early EEG in the ER, supporting the robustness of the electroclinical associations observed. Hyperperfusion on $\mathrm{pCT}$ had high sensitivity for predicting persistent continuous seizure activity compatible with SE on urgent EEG. The high negative predictive value observed suggests that the likelihood of observing SE on early EEG is very low in patients without hyperperfusion on $\mathrm{pCT}$. In addition, in the present study accompanying thalamic hyperperfusion was found to be a specific imaging marker of SE. In the first place, no single isolated seizure episode, even accompanied by cortical cerebral hyperperfusion, was associated to increased perfusion in thalamus. Moreover, as reported first on arterial spin labeling sequence on MRI by Ohtomo et al. [24] and recently on pCT by Giovannini et al. [22], thalamic hyperperfusion, although not sensitive, is highly specific of continuous sustained ictal activity compatible with SE on EEG. Therefore, the present study suggests that early pCT findings, together with other factors, such as clinical presentation [25], could serve as an adjunctive diagnostic tool for NCSE risk stratification; thus, probably avoiding delayed management in acute settings, especially if accompanied by the highly specific feature of thalamic hyperperfusion. 
Fig. 3 A 57-year-old man experienced a focal motoronset seizure that progressed to presumed non-convulsive status epilepticus without coma. Perfusion computed tomography performed within $2 \mathrm{~h}$ of symptom onset showed a focal increase in cerebral blood flow (A, arrow) together with a shortening of circulation times in the left frontal cortex (B and $\mathbf{C}$, arrows). All the above findings were accompanied by relative preservation of subcortical white matter and hyperperfused homolateral thalamus (D, arrow). An electroencephalogram acquired within $2 \mathrm{~h}$ of neuroimaging ( $\mathrm{E}$, ten seconds-epoch longitudinal bipolar montage) showed focal electrographic status epilepticus in the left fronto-central region without motor prominent symptoms. A second electroencephalogram performed $20 \mathrm{~h}$ later (F) showed left fronto-central periodic lateralized discharges without modifiers
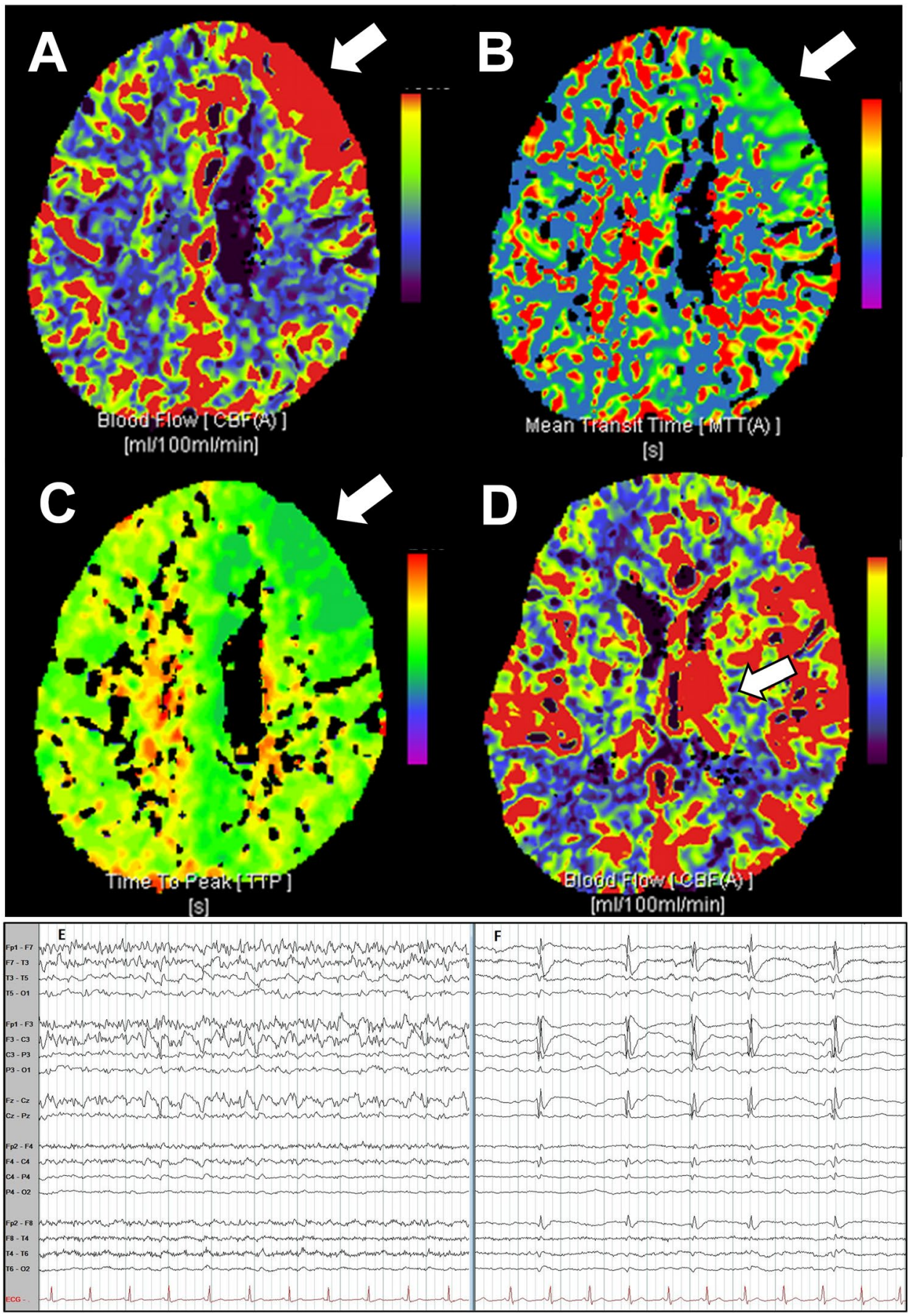

Reliable prospective data have shown that pCT performed within $80 \mathrm{~min}$ of the end of a seizure shows postictal hypoperfusion in $80 \%$ of patients with epilepsy [26]. In our series, however, pCT revealed nonspecific patterns-mainly normal brain perfusion-in patients diagnosed with a single epileptic seizure. Most retrospective studies assessing $\mathrm{pCT}$ changes in patients with single epileptic seizures in an acute setting have shown 
Table 2 Association between thalamic hyperperfusion and final diagnosis and EEG pattern

\begin{tabular}{|c|c|c|c|c|}
\hline & \multirow[t]{2}{*}{ Total } & \multicolumn{2}{|c|}{ Thalamic hyperperfusion } & \multirow[t]{2}{*}{$P$ value* } \\
\hline & & Yes $(n=8)$ & No $(n=39)$ & \\
\hline \multicolumn{5}{|c|}{ Final diagnosis } \\
\hline $\mathrm{SS} ; n(\%)$ & $27(57.4)$ & $0(0)$ & $27(69.2)$ & \multirow[t]{2}{*}{$<0.0001$} \\
\hline $\mathrm{SE} ; n(\%)$ & $20(42.6)$ & $8(100)$ & $12(30.8)$ & \\
\hline \multicolumn{5}{|l|}{$\mathrm{EEG}^{\ddagger}$ findings } \\
\hline $\mathrm{NEA} ; n(\%)$ & $19(46.3)$ & $0(0)$ & 19 (55.9) & \multirow[t]{4}{*}{0.003} \\
\hline IED; $n(\%)$ & $12(29.3)$ & $2(28.6)$ & $10(29.4)$ & \\
\hline SES; $n(\%)$ & $1(2.4)$ & $0(0)$ & $1(2.9)$ & \\
\hline $\mathrm{SE} ; n(\%)$ & $9(22)$ & $5(71.4)$ & $4(11.8)$ & \\
\hline
\end{tabular}

$S S$ singles seizure, $S E$ status epilepticus, $E E G$ electroencephalography, $N E A$ non-epileptiform abnormalities, IED interictal epileptiform discharges, SES single epileptic seizure

*Bold typed $p$ values represent statistically significant differences between groups.

\$: 41 patients underwent EEG registry. Percentages are calculated according to these numbers

similar results to ours [17]; thus, pCT seems not useful regarding the diagnostic process of single epileptic seizures. In addition, none of the patients with hypoperfusion on pCT in our study were diagnosed with SE. Other authors, however, have reported "ictal hypoperfusion" in a small number of cases that could possibly be explained by the presence of adjacent hyperperfused areas [27] or the acquisition of images during post-ictal periods [28]. In brief, the current evidence suggests that SE should not be contemplated in the differential diagnosis when $\mathrm{pCT}$ shows isolated hypoperfusion in a patient with focal neurological deficits in an ER setting.

We decided to use a visual rather than a quantitative approach $[29,30]$ to analyze the perfusion maps, as a previous study by our group showed that visual analysis appears to be more sensitive for the detection of hyperperfusion [9], and most importantly, is easily applicable to routine clinical practice.
The main limitations of our study are its small sample size and retrospective design, which inherently lead to biases, such as variations in the time of $\mathrm{pCT}$ and EEG acquisition and the interpretation of clinical features. To solve this issue, other group's studies included for analysis only those patients that underwent an EEG examination close in time after the pCT [20, 22]. However, in the present study patients were included if an EEG was performed within $24 \mathrm{~h}$ of symptom onset. Thus, probably capturing a more realistic picture of regular delayed conventional EEG performance in many ERs [5]. In addition, longer, continuous EEG recordings in the ER could improve the characterization of patients along the ictal-interictal continuum and its neuroimaging correlate obtained on pCT. Another limitation of our study is that we did not investigate the diagnostic specificity of pCT by comparing results between patients diagnosed with SE and controls with other conditions likely to cause cerebral hyperemia, such as encephalitis [31]. In addition, in light of the presented results, further studies could assess pCT imaging usefulness to differentiate between SE, and other acute presenting non-ictal mimics, such as confusional or dissociative psychogenic states. Nonetheless, our study brings new evidence and emphasizes the potential value of $\mathrm{pCT}$ in the assessment of epileptic disorders in ER settings.

\section{Conclusion}

Observation of focal brain hyperperfusion on $\mathrm{pCT}$ in an appropriate clinical setting should raise suspicion of SE and lead to further clinical and EEG investigation, especially if cerebral cortical hyperperfusion is accompanied by increased perfusion of homolateral thalamus. The absence of hyperperfusion rules out SE in most cases. pCT is not useful in the ER for the detection of single epileptic seizures in ER due to its low diagnostic yield. Larger, prospective studies are needed to further study $\mathrm{pCT}$ as an adjunctive diagnostic modality for NCSE and EEG findings along the ictal-interictal continuum in emergency settings.
Table 3 Predictive yield of cerebral cortical and thalamic hyperperfusion on $\mathrm{pCT}$ for SE as a final diagnosis and an SE pattern on EEG

\begin{tabular}{|c|c|c|c|c|}
\hline & \multicolumn{2}{|c|}{ Final diagnosis:status epilepticus (SE) } & \multicolumn{2}{|l|}{ EEG: SE pattern } \\
\hline & Cortical & Thalamic & Cortical & Thalamic \\
\hline $\mathrm{Se}, \%(95 \% \mathrm{CI})$ & $60(36.4-80.2)$ & $40(20-63.6)$ & $88.9(50.7-99.4)$ & $55.5(25.6-84.6)$ \\
\hline Sp, \% (95\% CI) & $77.8(57.2-90.6)$ & $100(84.5-100)$ & $75(56.2-87.9)$ & $93.7(77.8-98.9)$ \\
\hline PPV, \% (95\% CI) & $66.7(41.1-85.6)$ & $100(59.7-100)$ & $50(20.7-69.4)$ & $71.4(30.2-94.9)$ \\
\hline NPV \% (95\% CI) & $72.4(52.0-86.5)$ & $69.2(52.2-82.4)$ & $96(77.7-99.8)$ & $88.2(71.6-96.1)$ \\
\hline
\end{tabular}

$E E G$ electroencephalogram, $N P V$ negative predictive value, $P P V$ positive predictive value, $S e$ sensitivity, $S p$ specificity 
Acknowledgements This research did not receive a grant from funding agencies in the public, commercial, or not-for-profit sector. The authors thank Anne Murray for English language support.

Author contributions All authors contributed to the study conception and design. Material preparation, data collection and analysis were performed by Juan Luis Restrepo-Vera, Elena Fonseca, Pilar Coscojuela, Manuel Quintana, and Manuel Toledo. The first draft of the manuscript was written by Juan Luis Restrepo-Vera and all authors commented on previous versions of the manuscript. All authors read and approved the final manuscript.

Data availability The data that support the findings of this study are available from the corresponding author upon reasonable request.

\section{Declarations}

Conflicts of interest EF declares speaker fees from UCB Pharma, Esteve laboratorios, Eisai Inc and Sanofi Genzyme. ES declares speaker fees from UCB Pharma, BIAL Pharmaceutical, EISAI Inc. Arvelle and Esteve laboratorios. LA declares speaker fees from UCB Pharma, BIAL Pharmaceutical, EISAI Inc., Sanofi Genzyme and Esteve laboratorios. MT has received speaker honoraria from UCB Pharma, Esteve, BIAL Laboratories, and Sanofi. The other authors declare no financial or other conflicts of interest.

Ethical approval We confirm that we have read the Journal's position on issues concerning ethical publication and affirm that this report is consistent with those guidelines.

\section{References}

1. Rizos T, Jüttler E, Sykora $\mathrm{M}$ et al (2011) Common disorders in the neurological emergency room-experience at a tertiary care hospital. Eur J Neurol 18:430-435. https://doi.org/10.1111/j.14681331.2010.03170.x

2. Trinka E, Kälviäinen R (2017) 25 years of advances in the definition, classification and treatment of status epilepticus. Seizure 44:65-73. https://doi.org/10.1016/j.seizure.2016.11.001

3. Waterhouse EJ (2018) The epidemiology of status epilepticus. In: Drislane FW, Kaplan PW (eds) Status epilepticus, 2nd edn. Springer International Publishing, Cham, pp 15-30

4. Moulin S, Leys D (2019) Stroke mimics and chameleons. Curr Opin Neurol 32:54-59. https://doi.org/10.1097/WCO.0000000000 000620

5. Vespa PM, Olson DM, John S et al (2020) Evaluating the clinical impact of rapid response electroencephalography: the DECIDE multicenter prospective observational clinical study. Crit Care Med 48:1249-1257. https://doi.org/10.1097/CCM.0000000000 004428

6. Naylor DE, Liu H, Wasterlain CG (2005) Trafficking of GABA(A) receptors, loss of inhibition, and a mechanism for pharmacoresistance in status epilepticus. J Neurosci 25:7724-7733. https://doi. org/10.1523/JNEUROSCI.4944-04.2005

7. Sutter R, Kaplan PW, Rüegg S (2013) Outcome predictors for status epilepticus-what really counts. Nat Rev Neurol 9:525-534

8. Powers WJ, Rabinstein AA, Ackerson T et al (2018) 2018 Guidelines for the early management of patients with acute ischemic stroke: a guideline for healthcare professionals from the American Heart Association/American Stroke Association. Stroke 49:e46e110. https://doi.org/10.1161/STR.0000000000000158
9. González-Cuevas M, Coscojuela P, Santamarina E et al (2019) Usefulness of brain perfusion CT in focal-onset status epilepticus. Epilepsia 60:1317-1324. https://doi.org/10.1111/epi.16063

10. Strambo D, Rey V, Rossetti AO et al (2018) Perfusion-CT imaging in epileptic seizures. J Neurol 265:2972-2979. https://doi.org/10. 1007/s00415-018-9095-1

11. Van Cauwenberge MGA, Dekeyzer S, Nikoubashman O et al (2018) Can perfusion CT unmask postictal stroke mimics? A case-control study of 133 patients. Neurology 91:e1918-e1927. https://doi.org/10.1212/WNL.0000000000006501

12. Hogan-Cann AD, Lu P, Anderson CM (2019) Endothelial NMDA receptors mediate activity-dependent brain hemodynamic responses in mice. Proc Natl Acad Sci USA 116:10229-10231. https://doi.org/10.1073/pnas.1902647116

13. Mehra A, Guérit S, Macrez R et al (2020) Nonionotropic action of endothelial NMDA receptors on blood-brain barrier permeability via Rho/ROCK-mediated phosphorylation of myosin. J Neurosci 40:1778-1787. https://doi.org/10.1523/JNEUROSCI.0969-19. 2019

14. Trinka E, Cock H, Hesdorffer D et al (2015) A definition and classification of status epilepticus-report of the ILAE task force on classification of status epilepticus. Epilepsia 56:1515-1523. https://doi.org/10.1111/epi.13121

15. Hirsch LJ, LaRoche SM, Gaspard N et al (2013) American clinical neurophysiology society's standardized critical care EEG terminology: 2012 version. J Clin Neurophysiol 30:1-27. https://doi. org/10.1097/WNP.0b013e3182784729

16. Leitinger M, Beniczky S, Rohracher A et al (2015) Salzburg consensus criteria for non-convulsive status epilepticus-approach to clinical application. Epilepsy Behav 49:158-163. https://doi.org/ 10.1016/j.yebeh.2015.05.007

17. Gugger JJ, Llinas RH, Kaplan PW (2020) The role of CT perfusion in the evaluation of seizures, the post-ictal state, and status epilepticus. Epilepsy Res 159:106256. https://doi.org/10.1016/j. eplepsyres.2019.106256

18. López Ruiz R, Quintas S, Largo P et al (2019) Usefulness of multiparametric computerized tomography findings in the differential diagnosis of stroke mimics of epileptic origin: a preliminary study. Neurologia 34:73-79. https://doi.org/10.1016/j.nrl.2016.10.010

19. Subramaniam T, Jain A, Hall LT et al (2019) Lateralized periodic discharges frequency correlates with glucose metabolism. Neurology 92:e670-e674. https://doi.org/10.1212/WNL.0000000000 006903

20. Hauf M, Slotboom J, Nirkko A et al (2009) Cortical regional hyperperfusion in nonconvulsive status epilepticus measured by dynamic brain perfusion CT. AJNR Am J Neuroradiol 30:693698. https://doi.org/10.3174/ajnr.A1456

21. Van Paesschen W (2004) Ictal SPECT. Epilepsia 45(Suppl 4):3540. https://doi.org/10.1111/j.0013-9580.2004.04008.x

22. Giovannini G, Malagoli M, Turchi G et al (2021) Cortical and thalamic hyper-perfusion in non-convulsive status epilepticus. Relationship between perfusion CT patterns and Salzburg EEG criteria. Seizure 92:10-17. https://doi.org/10.1016/j.seizure.2021. 08.002

23. Payabvash S, Oswood M, Truwit C, Mckinney A (2015) Acute CT perfusion changes in seizure patients presenting to the emergency department with stroke-like symptoms: correlation with clinical and electroencephalography findings. Clin Radiol. https://doi.org/ 10.1016/j.crad.2015.06.078

24. Ohtomo S, Otsubo H, Arai $\mathrm{H}$ et al (2021) Hyperperfusion in the thalamus on arterial spin labelling indicates non-convulsive status epilepticus. Brain Commun. https://doi.org/10.1093/braincomms/ fcaa223

25. Requena M, Fonseca E, Olivé M et al (2019) The ADAN scale: a proposed scale for pre-hospital use to identify status epilepticus. Eur J Neurol 26:760-e55. https://doi.org/10.1111/ene.13885 
26. Li E, d'Esterre CD, Gaxiola-Valdez I et al (2019) CT perfusion measurement of postictal hypoperfusion: localization of the seizure onset zone and patterns of spread. Neuroradiology 61:9911010. https://doi.org/10.1007/s00234-019-02227-8

27. Lee HW, Hong SB, Tae WS (2000) Opposite ictal perfusion patterns of subtracted SPECT: Hyperperfusion and hypoperfusion. Brain 123(10):2150-2159. https://doi.org/10.1093/brain/123.10. 2150

28. Wiest R, von Bredow F, Schindler K et al (2006) Detection of regional blood perfusion changes in epileptic seizures with dynamic brain perfusion CT-a pilot study. Epilepsy Res 72:102110. https://doi.org/10.1016/j.eplepsyres.2006.07.017
29. Manganotti P, Furlanis G, Ajčević M et al (2019) CT perfusion and EEG patterns in patients with acute isolated aphasia in seizure-related stroke mimics. Seizure 71:110-115. https://doi.org/ 10.1016/j.seizure.2019.07.005

30. Austein F, Huhndorf M, Meyne J et al (2018) Advanced CT for diagnosis of seizure-related stroke mimics. Eur Radiol 28:17911800. https://doi.org/10.1007/s00330-017-5174-4

31. Li R, Shi P-A, Liu T-F et al (2019) Role of 3D pseudocontinuous arterial spin-labeling perfusion in the diagnosis and follow-up in patients with herpes simplex encephalitis. AJNR Am J Neuroradiol 40:1901-1907. https://doi.org/10.3174/ajnr.A6279 